\title{
OPTIMIZACIÓN DE LA REMOCIÓN SIMULTÁNEA DE NITRATO, NITRITO, AMONIO Y FOSFATO DE AGUAS RESIDUALES MUNICIPALES
}

\author{
Jerson Chuquimboques Marrero*a, Jorge Vergara Rojas ${ }^{\mathrm{a}}$, Jorge Mendoza Bobadilla*a,
}

\section{RESUMEN}

El presente estudio evalúa la capacidad de remoción del biochar de bagazo de caña de azúcar para nitrato, nitrito, amonio y fosfato presentes en aguas residuales municipales; asimismo, se determinó la dosis óptima $(\mathrm{mg} / \mathrm{ml})$ de biochar en la remoción sinérgica de estos contaminantes dentro de la matriz estudiada, para aquello se han desarrollado 10 tratamientos experimentales a niveles de dosis de biochar 0,$5 ; 1,0 ; 2,0 ; 3,0$ y 4,0 mg/ml y a tiempos de contactos de $2 \mathrm{~h}$ y $4 \mathrm{~h}$, los cuales se corrieron por triplicado y los porcentajes de remoción fueron presentados a través de su media y error estándar. Adicionalmente a la evaluación del efecto individual de la dosis de biochar y el tiempo de contacto, se realizó un ajuste de efectos bivariados a través de método de superficie respuesta y superposiciones de imágenes de contorno, donde se determinó que la zona de remoción máxima de 95,7 \%; 94,6 \%; 72,0 $\%$ y $57,0 \%$ para $\mathrm{NH}_{4}^{+}, \mathrm{PO}_{4}^{3-}, \mathrm{NO}_{2}{ }^{-}$y $\mathrm{NO}_{3}{ }^{-}$, respectivamente; se encuentra entre tiempo de contacto de 3,50 - 3,75 h y dosis de biochar 1,25-1,75 mg/ml. Finalmente la validación de las correlaciones se realizó a un nivel de significancia de $95 \%$, mostrando R2 de 93,01; 96,12; 96,15 y 57,59 \% para el ajuste de modelos de remoción bivariada para $\mathrm{NH}_{4}^{+}, \mathrm{PO}_{4}{ }^{3-}$, $\mathrm{NO}_{2}^{-}$- $\mathrm{NO}_{3}^{-}$, respectivamente.

Palabras clave: biochar, bagazo de caña, remoción simultánea, contaminantes de nitrógeno $\mathrm{y}$ fósforo, aguas residuales municipales.

\footnotetext{
a Laboratorio de Investigación en Aguas, Escuela de Ingeniería Ambiental, Universidad Nacional de Trujillo. Av. Juan Pablo II s/n - Ciudad Universitaria, Trujillo, Perú.

b Departamento Académico de Ingeniería Química, Facultad de Ingeniería Química, Universidad Nacional de Trujillo. Av. Juan Pablo II s/n - Ciudad Universitaria, Trujillo, Perú

* Autor correspondiente a: Laboratorio de Investigación en Aguas, Escuela de Ingeniería Ambiental, Universidad Nacional de Trujillo. Av. Juan Pablo II s/n-Ciudad Universitaria, Trujillo, Perú

E-mail address: jlmb76@hotmail.com (J. Mendoza)
} 


\title{
OPTIMIZATION SIMULTANEOUS REMOVAL OF NITRATE, NITRITE, AMMONIUM AND PHOSPHATE IN SEWAGE
}

\begin{abstract}
The present study evaluates the removal capacity of sugarcane bagasse biochar for nitrate, nitrite, ammonium and phosphate present in sewage; likewise, the optimum dose $(\mathrm{mg} / \mathrm{ml})$ of biochar was determined for the synergic elimination of these contaminants within the matrix studied, for which 10 experimental treatments have been developed at biochar dose levels of 0,$5 ; 1,0 ; 2,0 ; 3,0$ and $4,0 \mathrm{mg} / \mathrm{ml}$ and contact times of $2 \mathrm{~h}$ and $4 \mathrm{~h}$, which were performed in triplicate and the elimination percentages were presented through their mean and standard error. In addition to the evaluation of the individual effect of the biochar dose and the contact time, an adjustment of the bivariate effects was made through response superficies method and overlays of contour images, where it was determined that the maximum elimination zone was $95,7 \%$; 94,6 \%; 72,0 \% and 57,0 \% for $\mathrm{NH}_{4}^{+}, \mathrm{PO}_{4}^{3-}, \mathrm{NO}_{2}^{-}$y $\mathrm{NO}_{3}^{-}$, respectively; it is between the contact time of $3,50-3,75 \mathrm{~h}$ and the dose of biochar 1,25-1,75 mg/ml. Finally, the validation of the correlations was performed at a level of significance of $95 \%$, showing an R2 of 93,01; 96,12; 96,15 and 57,59\% for the adjustment of the bivariate elimination models for $\mathrm{NH}_{4}^{+}, \mathrm{PO}_{4}^{3-}, \mathrm{NO}_{2}^{-}$y $\mathrm{NO}_{3}^{-}$, respectively.
\end{abstract}

Key words: biochar, sugarcane bagasse, simultaneous removal, nitrogen and phosphorus contaminants, seawater.

\section{INTRODUCCIÓN}

La contaminación de las matrices de aguas por compuestos derivados del nitrógeno y fósforo se ha visto incrementada en los últimos años, a consecuencia de un tratamiento inadecuado en la eliminación y la falta de instrumentos ambientales que permitan reducir el impacto de las descargas de las aguas residuales domésticas que llevan estos contaminantes hacia los cuerpos de aguas. Por estas razones, la eliminación de los compuestos derivados de nitrógeno $(\mathrm{N})$ y fósforo $(\mathrm{P})$ de las aguas residuales se deben realizar para asegurar y garantizar la salud humana y el desarrollo sostenible.

El fósforo es considerado un elemento de suma importancia para la agricultura y la industria, pero su liberación excesiva en forma de fosfatos a los cuerpos de agua genera un proceso denominado eutrofización, el cual impone riesgos a los sistemas acuáticos y por ende genera pérdidas económicas. Hasta el momento, se han desarrollado métodos para la eliminación y recuperación de fosfatos que incluyen tratamiento biológico, precipitación química, adsorción, en la línea de líquidos y procesos termoquímicos en la línea de lodos. Para las técnicas de eliminación, la adsorción es considerada más útil y económica, especialmente para matrices de baja concentración de fosfatos como por ejemplo las aguas residuales domésticas ${ }^{1,2,3}$. 
La contaminación de los recursos hídricos por nitrógeno a través de sus formas iónicas (nitrato, nitrito y amonio) se ha convertido en un problema importante a nivel mundial en las últimas décadas, sin embargo el nitrógeno como recurso renovable es de gran importancia para el crecimiento de los organismos porque se puede ver que los principales compuestos nitrogenados involucrados en el ciclo del $\mathrm{N}$ incluyen nitrógeno gas $\left(\mathrm{N}_{2}\right)$, ion amonio $\left(\mathrm{NH}_{4}^{+}\right)$, nitrógeno orgánico, nitrato $\left(\mathrm{NO}_{3}^{-}\right)$y nitrito $\left(\mathrm{NO}_{2}^{-}\right)$. Se han presentado varios métodos que sirven para reducir estos compuestos del agua desde las técnicas biológicas que parece ser la más prometedora en el tratamiento de altas concentraciones, pero difícil para mantener sus condiciones óptimas. Sin embargo, la adsorción es un proceso muy factible para el tratamiento in situ de aguas subterráneas y superficiales, principalmente debido a su facilidad de aplicación, expresada en $\operatorname{costos}^{4,5}$.

El biochar, carbón vegetal producido a partir de la pirolisis de la biomasa, tiene una estructura única de poros y una superficie específica alta, que ha recibido mucha atención por la mejora del suelo, la gestión de residuos, la mitigación del cambio climático y la producción de energía ${ }^{5,6,8}$. El bagazo de caña de azúcar es el primer recurso de biomasa generado en la región La Libertad, como residuo de la fabricación del azúcar de caña; lo que se está evaluando su capacidad de remoción de contaminantes tales como orgánicos, metales pesados y otros de los cuerpos de agua. Este material adsorbente de bajo costo, producido a partir de los residuos de cultivos, por lo general tiene una menor capacidad de adsorción que las zeolitas y carbón activado; las investigaciones en los últimos años se han centrado en buscar materiales adsorbentes de diversos contaminantes en aguas de elevada eficiencia y rentables, optimizando sus variables de obtención del biochar y operación, por lo que se está buscando adsorbentes rentables para mejorar la eliminación de contaminantes como $\mathrm{NO}_{3}^{-}, \mathrm{NO}_{2}^{-}, \mathrm{NH}_{4}^{+}$ y $\mathrm{PO}_{4}^{3-}$ de las matrices de aguas contaminadas ${ }^{5,8}$.

El objetivo de este estudio fue optimizar las variables de adsorción simultanea de $\mathrm{NO}_{3}^{-}, \mathrm{NO}_{2}^{-}$, $\mathrm{NH}_{4}^{+}$y $\mathrm{PO}_{4}^{3-}$ en biochar obtenido de bagazo de caña; evaluando la correlación de la dosis de biochar y tiempo de contacto. Obteniendo la región donde la remoción simultánea de $\mathrm{NO}_{3}^{-}$, $\mathrm{NO}_{2}^{-}, \mathrm{NH}_{4}^{+}$y $\mathrm{PO}_{4}^{3-}$ es máxima, a través de modelos de optimización de procesos.

\section{PARTE EXPERIMENTAL}

\section{Materias primas}

Las muestras de agua residual fueron tomadas de los colectores principales de la Universidad Nacional de Trujillo (Facultad de Enfermería y a la llegada de la futura Planta de Tratamiento de Aguas), las cuales son producto de las actividades propias de la institución como son aguas sanitarias, los comedores y los generados por las actividades en los laboratorios tanto de investigación como de enseñanza. Respecto a la caracterización de las muestras se analizaron el $\mathrm{pH}$, conductividad eléctrica (uS/cm), y turbidez (NTU), siendo analizados a través del multiparámetro Termo Fisher Scientific; la concentración inicial de iones $\mathrm{NO}_{3}^{-}$,

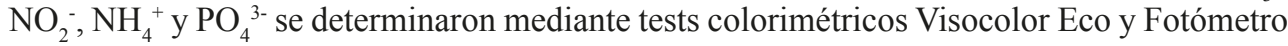
PF-12 Macharey Nagel en el Laboratorio de Investigación de Aguas (LIA) de la Universidad Nacional de Trujillo. 
En la elaboración de biochar se utilizó pulpa de bagazo de caña, considerado un residuo de biomasa ${ }^{6}$, procedente de la compactación del tallo de caña de azúcar en la extracción de guarapo (jugo de caña de azúcar). Se recolectaron muestras de bagazo de caña en los alrededores del Mercado Zonal Palermo de Trujillo, La Libertad, Perú.

\section{Pre-tratamiento de las materias primas}

En el punto de monitoreo se tomaron muestras de 5 litros cada hora desde las 8:00 am hasta las 4:00 p.m., conformando una muestra compuesta. Dejando sedimentar por espacio de 24 horas.

El bagazo de caña (biomasa) recolectado se cortó a tamaños $<40 \mathrm{~cm}$, lavados con agua desionizada y secados a condiciones naturales (corriente de aire y bajo sombra); posteriormente se trituró a tamaños $<8 \mathrm{~cm}$, secados en estufa a $105^{\circ} \mathrm{C}$ durante $24 \mathrm{~h}^{7,8}$.

La biomasa fue llevada a pirolisis bajo condiciones de oxígeno limitado en una cápsula de acero acoplada a un horno mufla, a una temperatura de $350^{\circ} \mathrm{C}$, durante un tiempo de calcinación de 3 horas (tratamiento térmico) ${ }^{8-10}$

El biochar obtenido fue sometido a proceso de molienda hasta tamaños $<2 \mathrm{~mm}$, teniendo como consecuencia un aumento del área superficial ${ }^{10}$.

\section{Ensayos de adsorción en sistema batch}

Se utilizaron dosis de biochar de: 0,$025 ; 0,050 ; 0,100 ; 0,150$ y $0,200 \mathrm{~g}$, en $50 \mathrm{ml}$ de muestra de agua residual, mediante agitación constante a $150 \mathrm{rpm}$ a temperatura ambiente $22 \pm 0,5$ ${ }^{\circ} \mathrm{C}$. Durante el proceso de adsorción de iones, se tomaron muestras a 2 y 4 horas de contacto, previo a los respectivos análisis de iones las muestras se filtraron.

Todos los análisis de adsorción se realizaron por triplicado, mostrando en los resultados el promedio de las réplicas.

\section{Análisis estadístico}

La caracterización fisicoquímica de las materias primas y los experimentos de remoción se realizaron por triplicado, y el error estándar se obtuvo mediante estadística descriptiva en ORIGIN PRO 9.1, al igual que el ajuste de los gráficos. Los modelos lineales y cuadráticos de optimización se ajustaron utilizando MATLAB 2018b, y se utilizaron los valores de R2 para comparar el rendimiento de los modelos. Las correlaciones se analizaron con la prueba de Pearson (bivariada) en $\mathrm{p}=0,05$ por INFOSTAT. 


\section{RESULTADOS Y DISCUSIÓN}

\section{Caracterización de las aguas residuales}

En la tabla 1 se presentan los valores de los parámetros analizados en la caracterización fisicoquímica de las muestras sedimentadas. Si bien el fósforo es uno de los nutrientes fundamentales para el desarrollo biológico, concentraciones elevadas de fosfatos en medios acuáticos provoca un fenómeno conocido como eutrofización, por ende, la concentración máxima de $\mathrm{PO}_{4}{ }^{3-}$ permitido en efluentes debe encontrarse en rangos de 2,0 a 0,1 ppm, en general, su alta concentración es vinculada con el uso frecuente de detergentes11, utilizados habitualmente en variadas formas de limpieza (materiales de laboratorio, ambientes de estudio, áreas de trabajo, entre otras) como se observa en la tabla 1, la concentración de $\mathrm{PO}_{4}{ }^{3-}$ se encuentra 20 veces por encima de los rangos anteriormente mencionados.

Tabla 1. Caracterización fisicoquímica de las muestras de experimentación

\begin{tabular}{|c|c|c|c|}
\hline Parámetros & $\begin{array}{c}\text { Facultad Enfermería } \\
\text { (AE) }\end{array}$ & $\begin{array}{c}\text { Futura PTAR } \\
\text { (AP) }\end{array}$ & $\begin{array}{l}\text { Agua para } \\
\text { experimentación a } \\
1(\mathrm{AE}): 1(\mathrm{AP})\end{array}$ \\
\hline $\mathrm{pH}$ & 8,00 & 8,15 & $8,06 \pm 0,036$ \\
\hline Turbiedad (NTU) & 1,46 & 2,63 & $1,97 \pm 0,036$ \\
\hline Conductividad $\left(\mathrm{mS} . \mathrm{cm}^{-1}\right)$ & 2,86 & 3,14 & $3,13 \pm 0,059$ \\
\hline Cianuro $\left(\mathrm{mg} . \mathrm{L}^{-1}\right)$ & 0,01 & $<0,01$ & $0,01 \pm 0,000$ \\
\hline Nitrito (mg. $\left.\mathrm{L}^{-1}\right)$ & 0,50 & 0,40 & $0,35 \pm 0,007$ \\
\hline Nitrato $\left(\mathrm{mg} . \mathrm{L}^{-1}\right)$ & 9,00 & 40,00 & $27,00 \pm 0,943$ \\
\hline Fosfato (mg.L-1 $)$ & 30,00 & 7,50 & $40,00 \pm 0,377$ \\
\hline Amonio (mg.L-1 $\left.{ }^{-1}\right)$ & 83,50 & 130,00 & $117,5 \pm 0,094$ \\
\hline
\end{tabular}

${ }^{\mathrm{a}}$ Valores son promedios \pm error estándar $(n=3)$.

En cuanto a los compuestos nitrogenados, se encuentran presentes dentro de un proceso de nitrificación, iniciando con el ion $\mathrm{NH}_{4}^{+}$, considerado como evidencia de reciente contaminación, su origen se debe principalmente a la materia orgánica presente en los desechos sanitarios cuya salida conecta directamente a la red de alcantarillado, donde se encuentran compuestos de nitrógeno presentes en la materia orgánica, respecto a los iones $\mathrm{NO}_{2}^{-}$y $\mathrm{NO}_{3}^{-}$, se forman mediante la trasformación del ion amonio, donde los desechos con presencia de nitrógeno orgánico se degradan formando amoniaco, el cual en medios acuosos reacciona con iones hidronio transformándose en iones amonio posteriormente, mediante bacterias autótrofas aerobias, son oxidados formando iones nitritos, los cuales al oxidarse nuevamente dan origen a iones nitratos finalizando el proceso de nitrificación ${ }^{12}$.

\section{Efecto de la dosis de biochar y el tiempo de contacto}

En la figura 1 se presentan los valores de los porcentajes de remoción de los iones en estudio, en función de la dosificación y tiempo de contacto para cada tratamiento. 


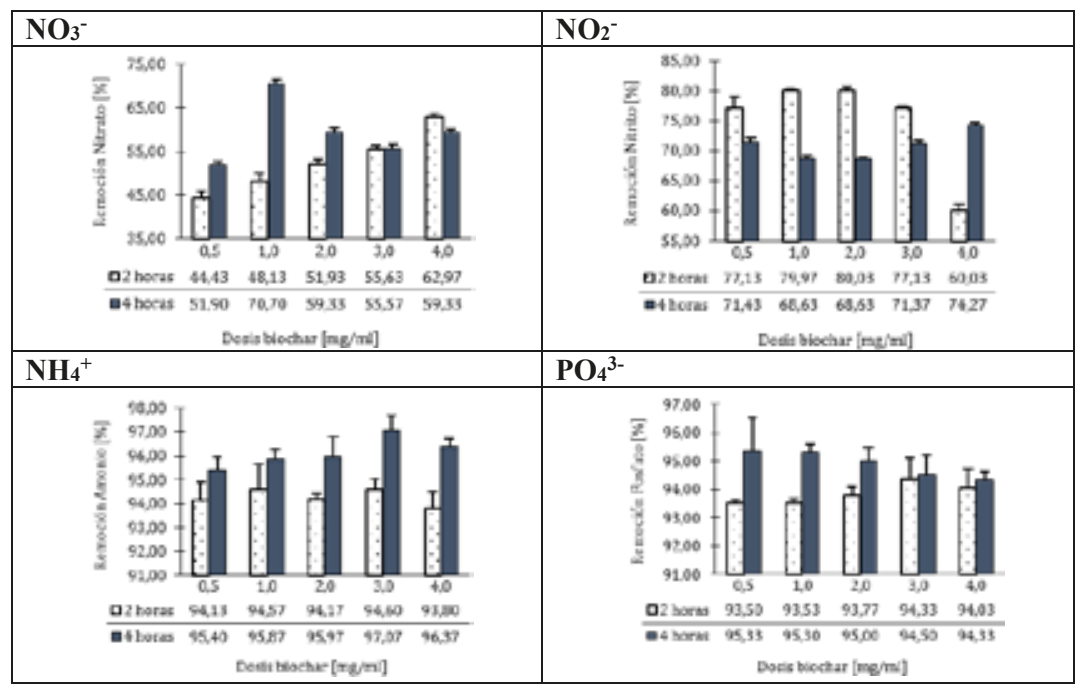

Figura 1. Evaluación de la dosis de biochar $[\mathrm{mg} / \mathrm{ml}]$ en el porcentaje de remoción de

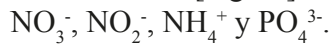

En la figura 1 se observa que los mejores porcentajes de remoción se obtuvieron en un tiempo de contacto (TC) de 4 horas con la excepción del ión $\mathrm{NO}_{2}^{-}$, lográndose una remoción mayor al $90 \%$ para $\mathrm{PO}_{4}^{3-}$ y $\mathrm{NH}_{4}^{+}$; puesto que se ha demostrado que estos iones presentan una mejor eficiencia en su remoción en comparación con otros ${ }^{13,14}$. La figura 2 demuestra que la capacidad de remoción de $\mathrm{PO}_{4}{ }^{3-}$ y $\mathrm{NH}_{4}{ }^{+}$no se ve influenciada por la dosis de biochar ni por el TC en los rangos estudiados; puesto que al aumentar la dosis de biochar y el TC no hubo una mejora significativa en la remoción de iones. $\mathrm{El} \mathrm{NH}_{4}^{+}$logra alcanzar su mayor porcentaje de remoción en un TC de $1 \mathrm{~h}$ utilizando Biochar producido a partir de aserrín de roble ${ }^{13}$.

$2 h$

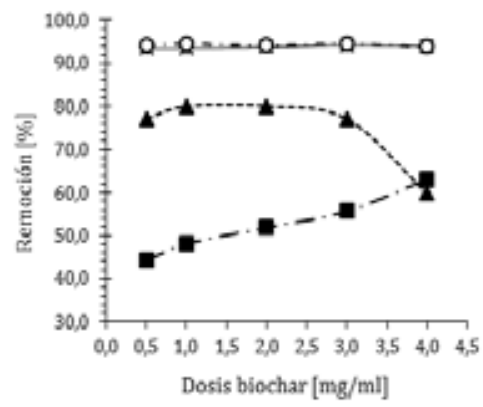

4h

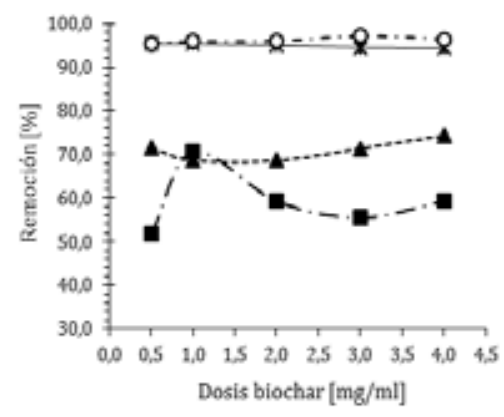

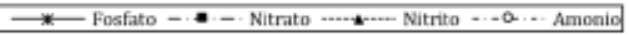

Figura 2. Análisis comparativo del porcentaje de remoción de $\mathrm{NO}_{3}^{-}, \mathrm{NO}_{2}^{-}, \mathrm{NH}_{4}^{+}$y $\mathrm{PO}_{4}^{3-}$ a tiempos de contacto de $2 \mathrm{~h}$ y $4 \mathrm{~h}$. 
$\mathbf{A}$
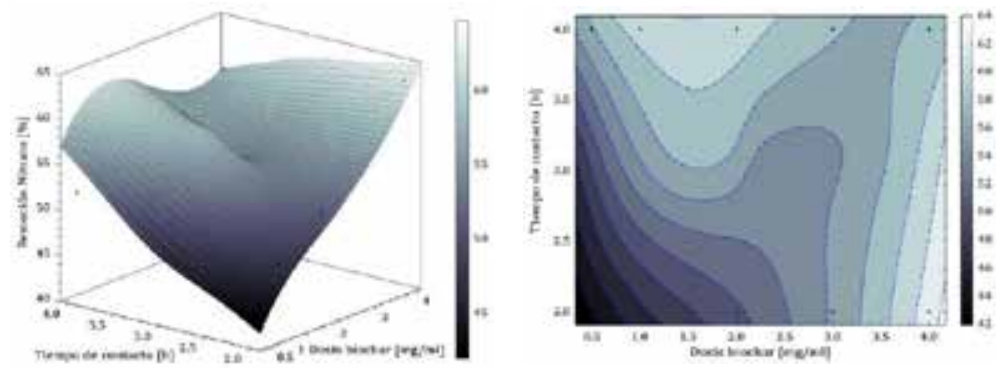

B
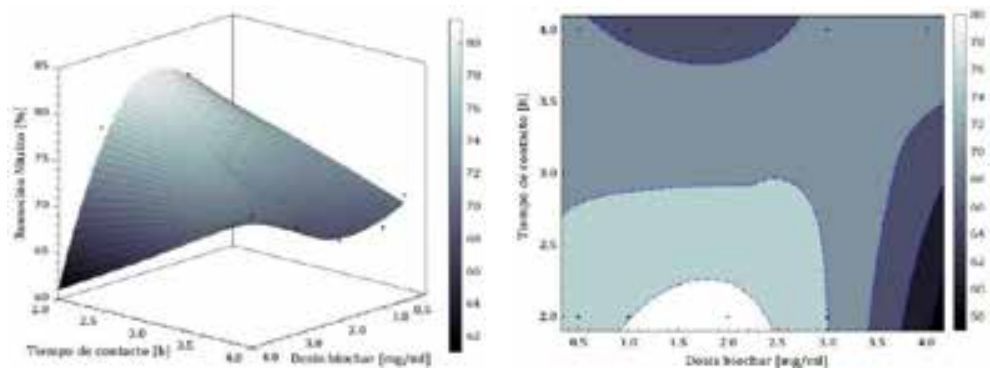

C
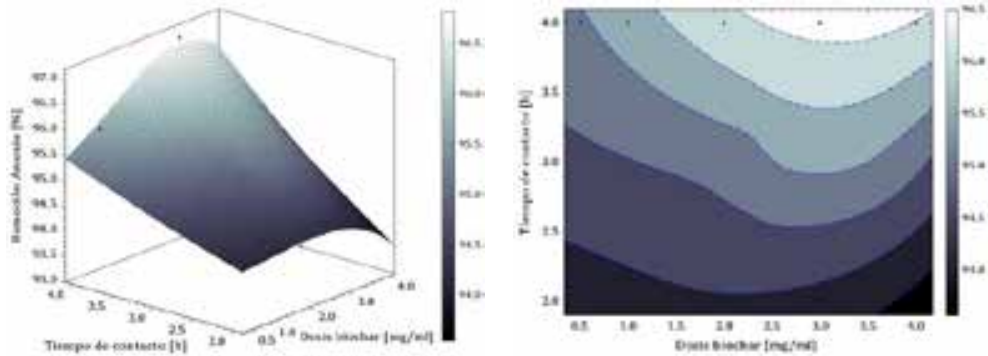

D
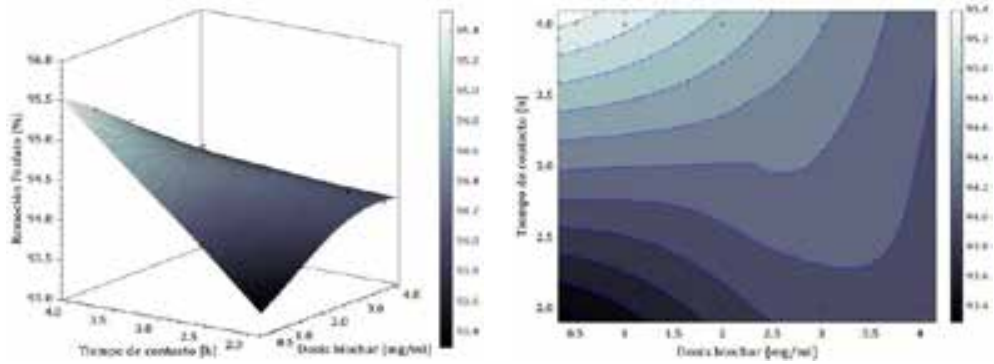

Figura 3. Gráficos de superficie $3 \mathrm{D}$ y contorno $2 \mathrm{D}$ para remoción de (a) $\mathrm{NO}_{3}^{-}$, (b) $\mathrm{NO}_{2}^{-}$, (c) $\mathrm{NH}_{4}^{+}$y (d) $\mathrm{PO}_{4}^{3-}$ a diferente dosificación de biochar y tiempo de contacto. 
Analizando la figura 3, se observa que los mejores porcentajes de remoción del ión nitrato $\mathrm{NO}_{3}^{-}$, se obtienen a dosis altas de biochar en TC bajos y a dosis bajas necesitan TC altos, debido a la baja concentración inicial que presentan estos tipos de aguas residuales.

Respecto al ion $\mathrm{NO}_{2}^{-}$se observa una mejor remoción a TC cortos, debido a las bajas concentraciones presentes en la muestra de ARD de esta manera; a tiempos $<3 \mathrm{~h}$ y dosificaciones inferiores a $3,0 \mathrm{mg} / \mathrm{ml}$ se obtienen los mejores resultados; sin embargo, al aumentar el TC se observa una disminución del porcentaje de remoción, lo que se debe a la oxidación de $\mathrm{NH}_{4}^{+}$generando iones nitritos, provocando un aumento en su concentración.

Para el $\mathrm{PO}_{4}{ }^{3-} \mathrm{y} \mathrm{NH}_{4}{ }^{+}$se observa que los mejores porcentajes de remoción se presentan en tiempos de contacto mayores a 3 horas y dosis de biochar superiores a $1 \mathrm{mg} / \mathrm{ml}$ para el $\mathrm{NH} 4+$ y menores a $2,5 \mathrm{mg} / \mathrm{ml}$ para el $\mathrm{PO}_{4}{ }^{3}$; como se muestra en la tabla 1 , el $\mathrm{NH}_{4}^{+}$es el ion de mayor concentración que presenta las aguas residuales, por tal motivo necesita mayores concentraciones para una mejor remoción.

La tabla 2 presenta las soluciones de la predicción de respuesta múltiple para la remoción de los iones, donde se evaluó cinco posibles soluciones y se verificó que el mejor tiempo de contacto para obtener los mejores porcentajes de remoción es de 4h; sin embargo, se encontró que $1 \mathrm{mg} / \mathrm{ml}$ es la mejor dosis de biochar obteniendo una deseabilidad compuesta del 71,9\% de la remoción de los iones estudiados; también se encontró que $4 \mathrm{mg} / \mathrm{ml}$ de dosis de biochar a 4 h de tiempo de contacto nos da una deseabilidad compuesta del 61,5\% de remoción; pero en términos de rentabilidad para la aplicación de biochar en la remoción de iones, las condiciones de la solución 1 son las deseables para esta aplicación ${ }^{15}$.

Tabla 2. Soluciones de la predicción de respuesta múltiple para la remoción de $\mathrm{NO}_{3}^{-}, \mathrm{NO}_{2}^{-}, \mathrm{NH}_{4}^{+}$y $\mathrm{PO}_{4}^{3-}$.

\begin{tabular}{|c|c|c|c|c|c|c|c|}
\hline \multirow[b]{2}{*}{ Solución } & \multirow{2}{*}{$\begin{array}{l}\text { Tiempo de } \\
\text { contacto }[\mathrm{h}]\end{array}$} & \multirow{2}{*}{$\begin{array}{l}\text { Dosis } \\
\text { biochar }[\mathrm{mg} / \mathrm{ml}]\end{array}$} & \multicolumn{4}{|c|}{ Remoción [\%] } & \multirow{2}{*}{$\begin{array}{l}\text { Deseabilidad } \\
\text { compuesta }\end{array}$} \\
\hline & & & $\mathrm{NH}_{4}{ }^{+}$ & $\mathrm{NO}_{2}^{-}$ & $\mathrm{NO}_{3}^{-}$ & $\mathrm{PO}_{4}{ }^{3-}$ & \\
\hline 1 & 4 & 1 & 95,87 & 68,63 & 70,70 & 95,30 & 0,71933 \\
\hline 2 & 4 & 4 & 96,37 & 74,27 & 59,33 & 94,33 & 0,61596 \\
\hline 3 & 4 & 2 & 95,97 & 68,63 & 59,33 & 95,00 & 0,60351 \\
\hline 4 & 4 & 3 & 97,07 & 71,37 & 55,57 & 94,50 & 0,60206 \\
\hline 5 & 4 & 0,5 & 95,40 & 71,43 & 51,90 & 95,33 & 0,53067 \\
\hline
\end{tabular}

La figura 4 presenta la región óptima de remoción de los iones por superposición de gráficas de contornos, siendo la región estimada en un tiempo de contacto entre $3,5-3,8 \mathrm{~h}$ y dosis de biochar $1,25-1,75 \mathrm{mg} / \mathrm{ml}$.

Por lo tanto, en base a los resultados de este estudio, se recomienda una dosis de adsorción de $1,5 \mathrm{mg} / \mathrm{ml}$ durante un tiempo de contacto de 3,6 h para experimentos de remoción múltiple. Con la dosificación y tiempo de contacto mencionado, se encontrará un equilibrio en la remoción de los iones del 95,7 \%; 94,6 \%; 72,0 \% y 57,0\% para $\mathrm{NH}_{4}^{+}, \mathrm{PO}_{4}{ }^{3-}, \mathrm{NO}_{2}^{-}$y $\mathrm{NO}_{3}^{-}$, respectivamente. 


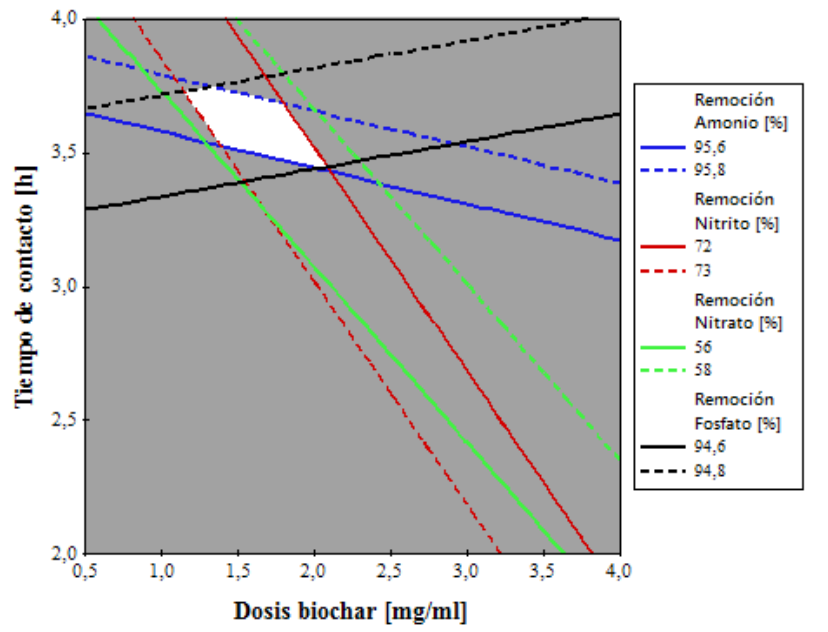

Figura 4. Superposición de gráficas de contornos $2 \mathrm{D}$ de regiones con alto potencial de remoción para $\mathrm{NO}_{3}^{-}, \mathrm{NO}_{2}^{-}, \mathrm{NH}_{4}^{+}$y $\mathrm{PO}_{4}^{3-}$.

\section{Análisis del ajuste de los efectos lineales y cuadráticos sobre la remoción}

Se ha realizado un ajuste de modelos con efecto lineal y cuadrático sobre las variables de estudios (dosis de biochar y el tiempo de contacto) para poder optimizar la remoción de $\mathrm{NO}_{3}^{-}, \mathrm{NO}_{2}^{-}, \mathrm{NH}_{4}^{+}$y $\mathrm{PO}_{4}^{3-}$. La tabla 3 presenta el resumen estadístico del ajuste de modelos, se muestra solamente que el modelo depende del tiempo de contacto sobre la remoción de fosfato y amonio su ajuste explica el 64,11 \% y 78,90 \% de la variación total en los datos sobre el promedio, respectivamente. Asimismo, estos modelos son estadísticamente signicativos a nivel de probabilidad de $95 \%$, mostrado un $p=0,003$ para fosfato y un $p=$ 0,000 para amonio. Por lo tanto, se interpreta que el aumento del tiempo de contacto tiene efectos crecientes sobre la remoción de estos contaminantes. Adicionalmente, para mejorar y optimizar la remoción simultanea de $\mathrm{NO}_{3}^{-}, \mathrm{NO}_{2}^{-}, \mathrm{NH}_{4}^{+}$y $\mathrm{PO}_{4}^{3-}$, se ajustaron modelos cuadráticos que expliquen la mejora de las interacciones bivariadas sobre la remoción; se demostró que el ajuste de los modelos sobre la remoción mejora cuando se estudian efectos bivariados, porque explican alrededor del $95 \%$ de la variación de la data. El nitrito, fosfato y amonio presentan un $\mathrm{R}^{2}$ de 96,15 \%; 96,12 \% y 93,01 \% que explica la validación del modelo para la optimización del modelo; sin embargo, el modelo del nitrito es solo explicado por un $57,59 \%$ de la variación total de la data. 
Tabla 3. S Resumen estadístico del ajuste de regresión lineal y cuadrática para la optimización de la remoción de $\mathrm{NO}_{3}^{-}, \mathrm{NO}_{2}^{-}, \mathrm{NH}_{4}^{+}$y $\mathrm{PO}_{4}^{3-}$.

\begin{tabular}{|c|c|c|c|c|c|c|c|c|}
\hline \multicolumn{9}{|c|}{ Fosfato $\left(\mathrm{PO}_{4}{ }^{3-}\right)$} \\
\hline \multirow{2}{*}{ Efecto } & \multirow{2}{*}{ GL } & \multirow{2}{*}{ SC Ajust. } & \multirow{2}{*}{ MSE } & \multirow{2}{*}{ Valor F } & \multirow{2}{*}{ Valor $\mathrm{p}$} & \multicolumn{3}{|c|}{ Resumen del modelo } \\
\hline & & & & & & RMSE & R-cuad. & R-cuad Ajust. \\
\hline $\mathrm{A}$ & 1 & 0,00028 & 0,00028 & 17,08 & $0,003^{*}$ & 0,00406 & $68,10 \%$ & $64,11 \%$ \\
\hline $\mathrm{B}$ & 4 & 0,00001 & 0,00000 & 0,03 & 0,997 & 0,00897 & $2,42 \%$ & $0,00 \%$ \\
\hline $\mathrm{A}: \mathrm{B}$ & 6 & 4,03543 & 0,01785 & & & 0,13360 & $97,41 \%$ & $96,12 \%$ \\
\hline \multicolumn{9}{|c|}{ Nitrato $\left(\mathrm{NO}_{3}{ }^{-}\right)$} \\
\hline \multirow{2}{*}{ Efecto } & \multirow{2}{*}{ GL } & \multirow{2}{*}{ SC Ajust. } & \multirow{2}{*}{ MSE } & \multirow{2}{*}{ Valor F } & \multirow{2}{*}{ Valor $\mathrm{p}$} & \multicolumn{3}{|c|}{ Resumen del modelo } \\
\hline & & & & & & RMSE & R-cuad. & R-cuad Ajust \\
\hline $\mathrm{A}$ & 1 & 0,01142 & 0,01142 & 2,26 & 0,171 & 0,07102 & $22,06 \%$ & $12,32 \%$ \\
\hline $\mathrm{B}$ & 4 & 0,02000 & 0,00500 & 0,79 & 0,580 & 0,07972 & $38,64 \%$ & $0,00 \%$ \\
\hline $\mathrm{A}: \mathrm{B}$ & 6 & 370,23123 & 24,32619 & & & 4,93216 & $71,72 \%$ & $57,59 \%$ \\
\hline \multicolumn{9}{|c|}{ Nitrito $\left(\mathrm{NO}_{2}{ }^{-}\right)$} \\
\hline \multirow{2}{*}{ Efecto } & \multirow{2}{*}{ GL } & \multirow{2}{*}{ SC Ajust. } & \multirow{2}{*}{ MSE } & \multirow{2}{*}{ Valor F } & \multirow{2}{*}{ Valor $\mathrm{p}$} & \multicolumn{3}{|c|}{ Resumen del modelo } \\
\hline & & & & & & RMSE & R-cuad. & R-cuad Ajust. \\
\hline $\mathrm{A}$ & 1 & 0,00396 & 0,00396 & 1,03 & 0,339 & 0,06188 & $11,45 \%$ & $0,38 \%$ \\
\hline $\mathrm{B}$ & 4 & 0,00812 & 0,00203 & 0,38 & 0,813 & 0,07276 & $23,48 \%$ & $0,00 \%$ \\
\hline $\mathrm{A}: \mathrm{B}$ & 6 & 336,12423 & 1,47599 & & & 1,21490 & $97,43 \%$ & $96,15 \%$ \\
\hline \multicolumn{9}{|c|}{$\operatorname{Amonio}\left(\mathrm{NH}_{4}{ }^{+}\right)$} \\
\hline \multirow{2}{*}{ Efecto } & \multirow{2}{*}{ GL } & \multirow{2}{*}{ SC Ajust. } & \multirow{2}{*}{ MSE } & \multirow{2}{*}{ Valor F } & \multirow{2}{*}{ Valor $\mathrm{p}$} & \multicolumn{3}{|c|}{ Resumen del modelo } \\
\hline & & & & & & RMSE & R-cuad. & R-cuad Ajust. \\
\hline $\mathrm{A}$ & 1 & 0,00090 & 0,00090 & 34,64 & $0,000^{*}$ & 0,00510 & $81,24 \%$ & $78,90 \%$ \\
\hline $\mathrm{B}$ & 4 & 0,00013 & 0,00003 & 0,16 & 0,947 & 0,01401 & $11,65 \%$ & $0 ., 0 \%$ \\
\hline $\mathrm{A}: \mathrm{B}$ & 6 & 10,34009 & 0,08422 & & & 0,29021 & $95,34 \%$ & $93,01 \%$ \\
\hline
\end{tabular}

* Significativo en el nivel de probabilidad $0_{2} .05$

Nota. A: Tiempo de contacto, B: Dosis biochar

\section{CONCLUSIONES}

El biochar de bagazo de caña de azúcar es un adsorbente verde y de bajo costo en el campo de la remediación ambiental, demostrando gran potencial en la remoción de contaminantes de las aguas residuales municipales, principalmente en $\mathrm{NH}_{4}^{+}$y $\mathrm{PO}_{4}^{3-}$, obteniéndose porcentajes de remoción mayores al $90 \%$; el uso de biochar como adsorbente proporciona un medio económicamente viable para tratar la biomasa residual.

Las condiciones óptimas de remoción simultánea de $\mathrm{NO}_{3}^{-}, \mathrm{NO}_{2}^{-}, \mathrm{NH}_{4}^{+}$y $\mathrm{PO}_{4}^{3-}$ de las aguas residuales municipales utilizando biochar a partir de bagazo de caña de azúcar se encuentra en un tiempo de contacto entre $3,5-3,8 \mathrm{~h}$ y dosis de biochar entre $1,25-1,75 \mathrm{mg} / \mathrm{ml}$.

\section{AGRADECIMIENTO}

Al Laboratorio de Investigación en Aguas "LIA" de la Escuela de Ingeniería Ambiental de la Universidad Nacional de Trujillo. 


\section{REFERENCIAS BIBLIOGRÁFICAS}

1. Ye Y, Ngo HH, Guo W, Liu Y, Li J, Liu Y, et al. Insight into chemical phosphate recovery from municipal wastewater. Sci Total Environ. 2017; 576: 159-171.

2. Cui X, Dai X, Khan KY; Li T, Yang X, He Z. Removal of phosphate from aqueous solution using magnesium-alginate/chitosan modified biochar microspheres derived from Thalia dealbata. Bioresour Technol. 2016; 218:1123-1132.

3. Yu P, Xue Y, Gao F, Liu Z, Cheng X, Yang K. Phosphorus Removal from Aqueous Solution by Pre- or Post-Modified Biochars Derived from Agricultural Residues. Water Air Soil Pollut. 2016; 227:370. doi: 10.1007/s11270-016-3066-X

4. Mizuta K, Matsumoto T, Hatate Y, Nishihara K, Nakanishi T. Removal of nitratenitrogen from drinking water using bamboo powder charcoal. Bioresour Technol. 2004; 95 (3): 255-257.

5. Ye Y, Huu Hao N, Guo W, Liu Y, Chang SW, Dinh Duc N, et al. A critical review on ammonium recovery from wastewater for sustainable wastewater management. Bioresour Technol. 2018; 268: 749-758.

6. Hafshejani LD, Hooshmand A, Naseri AA, Mohammadi AS, Abbasi F, Bhatnagar A. Removal of nitrate from aqueous solution by modified sugarcane bagasse biochar. Ecol Eng. 2016, 95: 101-111.

7. Rice EW, Baird RB, Eaton AD, Clesceri LS. Standard Methods for the Examination of Water and Wastewater. 22nd Edition ed. Washington: American Water Works Association, Water Environment Federation: 2012; p 1496.

8. Kameyama K, Miyamoto T, Shiono T, Shinogi Y. Influence of Sugarcane Bagassederived Biochar Application on Nitrate Leaching in Calcaric Dark Red Soil. J Environ Qual. 2012; 41 (4): 1131-1137.

9. Jung K-W, Jeong T-U, Kang H-J, Chang J-S, Ahn K-H. Preparation of modified-biochar from Laminaria japonica: Simultaneous optimization of aluminum electrode-based electro-modification and pyrolysis processes and its application for phosphate removal. Bioresour Technol. 2016; 214: 548-557.

10. Lou K, Rajapaksha AU, Ok YS, Chang SX. Pyrolysis temperature and steam activation effects on sorption of phosphate on pine sawdust biochars in aqueous solutions. Chem Spec Bioavailab. 2016; 28(1-4): 42-50.

11. Rueda R, De los Santos A, Fuentes A, Gutiérrez G. Toxicidad letal y subletal del fosfato de sodio dibásico y efectos en branquias y conducta de las crías del pez goodeido Skiffia multipunctata. Hidrobiología. 2014; 24 (3): 207-214.

12. Sardiñas O, Pérez A. Determinación de nitrógeno amoniacal y total en aguas de consumo y residuales por el método del fenato. [Internet]. Rev Cubana Hig Epidemiol. 2004; 42 (2). [Citado 12 set 2018] Disponible en: http://scielo.sld.cu/scielo.php?script=sci_ arttext\&pid $=$ S1561-30032004000200002

13. Wang Z, Guo H, Shen F, Yang G, Zhang Y, Zeng Y, et al. Biochar produced from oak sawdust by Lanthanum (La)-involved pyrolysis for adsorption of ammonium (NH4+ ), nitrate (NO3-), and phosphate (PO43- ). Chemosphere. 2015; 119: 646-653. 
14. Bock E, Smith N, Rogers M, Coleman B, Reiter M, Benham, B. et al. Enhanced Nitrate and Phosphate Removal in a Denitrifying Bioreactor with Biochar. J Environ Qual. 2015; 44(2): 605-613.

15. Yin Q, Wang R, Zhao Z. Application of Mg-Al-modified biochar for simultaneous removal of ammonium, nitrate, and phosphate from eutrophic water. J Clean Prod. 2018; 176: 230-240. 